\title{
Estimation-Based Search Space Traversal in PILP Environments
}

\author{
Joana Côrte-Real, Inês Dutra, and Ricardo Rocha \\ CRACS \& INESC TEC and Faculty of Sciences, University of Porto \\ Rua do Campo Alegre, 1021/1055, 4169-007 Porto, Portugal \\ $\{\mathrm{jcr}$, ines, ricroc\}@dcc.fc.up.pt
}

\begin{abstract}
Probabilistic Inductive Logic Programming (PILP) systems extend ILP by allowing the world to be represented using probabilistic facts and rules, and by learning probabilistic theories that can be used to make predictions. However, such systems can be inefficient both due to the large search space inherited from the ILP algorithm and to the probabilistic evaluation needed whenever a new candidate theory is generated. To address the latter issue, this work introduces probability estimators aimed at improving the efficiency of PILP systems. An estimator can avoid the computational cost of probabilistic theory evaluation by providing an estimate of the value of the combination of two subtheories. Experiments are performed on three real-world datasets of different areas (biology, medical and web-based) and show that, by reducing the number of theories to be evaluated, the estimators can significantly shorten the execution time without losing probabilistic accuracy.
\end{abstract}

\section{Introduction}

Probabilistic Inductive Logic Programming (PILP) [4] is an extension of the ILP paradigm that can represent knowledge using probabilistic facts and rules and which learns, as a result, probabilistic theories that can be used for prediction. Introducing probabilistic information in ILP to create PILP can be used to (i) create better logical models that can take uncertainty into account; (ii) implicitly reduce the theory search space by transforming numerical arguments in annotated probabilistic data; (iii) compress data by representing it as aggregates; or (iv) add knowledge from the literature in the form of probabilistic information. PILP can be seen as a Statistical Relational Learning approach (SRL) [4] and, in this setting, both parameter and structure learning are possible. However, it is more common for SRL techniques to learn parameters, and only few SRL methods can learn structure, or both. PILP differs from other SRL techniques because it focuses primarily on structure learning over relational data that is already annotated with probabilistic values.

PILP suffers from the same search space traversal efficiency issues as ILP because similar algorithms are used to generate the logical part of the theories. Additionally, PILP adds a level of complexity because every new theory generated needs to be probabilistically evaluated in order to be considered. This 
work presents a strategy aimed at improving the performance of PILP systems through the use of estimators that can prune the universe of candidate theories and, thus, reduce the search space. These estimators were integrated in the SkILL system [2], but the concepts are general to any PILP engine.

SkILL is a stochastic inductive logic learner which can generate First-Order Logic (FOL) theories based on a database of probabilistic data. These theories are expressed as Horn clauses (a subset of FOL) and so they can be used to extract relational non-trivial knowledge about the dataset where they are inferred from. SkILL differs from other PILP systems such as ProbFOIL+ [3] or SLIPCOVER [1] because it introduces an algorithm of polynomially bound complexity on userdefined parameters, as well as a number of efficient pruning strategies that can reduce execution time while maintaining prediction quality.

To the best of the authors' knowledge, the notion of an estimator is a novel feature in PILP systems. In this work, five estimators that can be incorporated in the estimation pruning strategy are proposed, namely minimum, maximum, center, independence and exclusion. To validate this estimation-based search space traversal approach, a thorough experimental analysis of the impact that each estimator has on the execution time and theory quality is presented. Experiments are performed in three probabilistic datasets, and the models are validated using hold-out resampling or leave-one-out cross-validation techniques. Results show that estimators can significantly prune the search space, and thus, reduce execution time, while maintaining the same probabilistic accuracy when compared with using no estimation pruning.

\section{Related Work}

According to Getoor et al. [8], relational data introduces the machine learning problem of class-level frequency estimation: building a model that can answer generic statistical queries about classes of individuals in a database. This is opposed to instance-level frequency estimation, where one is interested in the probability of a particular instance. In a first-order logic representation, the first type of estimation would be described with first-order formulas with variables, while the second type would be described with first-order formulas with constants (ground terms). There has been a whole body of research on modeling relational data using various kinds of representations, inference systems, and learning techniques (parameters and structure) [7].

There are many probabilistic languages that can represent and perform inference with probabilities, such as SLP [12], BLP [9], CLP(BN) [15], ProbLog [10], MLN [14], Prism [16], among others (for a recent survey of probabilistic logic languages see [5]). Probabilistic logic languages have been around for over 20 years [5]. They differ in the way they extend logic to include probabilities (class-level or instance-level), in their syntax, in the kind of uncertainty that is represented (probabilities, weights or potential functions), and in their inference algorithms. However, there are few works in the probabilistic logic field 
dedicated to learning the structure of classifiers using a human-readable probabilistic representation for knowledge.

An example of a system that uses logic to learn from probabilistic representations is MLN, where the learning algorithm is ILP-based and uncertainty is represented as potential functions [11]. Several methods of inference can be used and the models learnt are first-order logic formulas with potential scores. Another ILP-based example is Natarajan et al.'s boosting approach [13], which uses regression trees to learn the model structure faster. Furthermore, some works in the literature allow the representation of probabilistic logic using Bayesian networks. This is the case of Schulte et al.'s PBN (Parametrized Bayesian Networks) [17]. PBN is a first-order logic extension of Bayesian networks, where nodes are represented as a first-order term with a variable.

This work's focus is on probabilistic inductive logic programming (PILP) systems. These systems use as basis a probabilistic logic language to learn (probabilistic) theories. This work follows the syntax and inference mechanism of ProbLog [10], and uses it to represent the datasets and to learn first-order (probabilistic) theories. ProbLog is an extension of Prolog, whose syntax is modified to take into account class-level and instance-level probabilities, and annotated disjunctions. Uncertainty is, thus, represented as probabilities.

There are several PILP approaches mentioned in the literature, such as ProbFOIL/ProbFOIL+ [3], SLIPCOVER [1] and SkILL [2]. ProbFOIL+ is a PILP system that can tune the prediction of a theory by finding a weight for each rule in that theory. ProbFOIL+ algorithm computes the best weight whenever a rule is being added to the theory and then integrates it in the theory. This can be seen as a form of boosting, since the importance of each rule in the theory is being adjusted, even though the possibility for adjustment is limited (the weight must be between 0 and 1). SLIPCOVER introduces the new ability to perform generative learning in the search space. SLIPCOVER still requires a target predicate, but it also gathers a set of good theories which can explain predicates from the BK other than the target predicate - this process can be viewed as a form of deep learning, since these intermediate theories will be used to explain the target predicate. SkILL is a PILP system which introduces an algorithm of polynomially bound complexity on user-defined parameters, as well as a number of efficient pruning strategies that can reduce execution time while maintaining prediction quality. A comparison between SkILL, ProbFOIL+ and an ILP system can be found in [2].

\section{Background}

PILP extends the ILP setting by introducing Probabilistic Background Knowledge (PBK), where FOL data descriptions can be annotated with a probability value ranging from 0 to 1, and Probabilistic Examples (PE), no longer positive or negative, also with a value ranging between 0 and 1 . Because PILP theories are still generated based on the logical information of the data, the ILP language bias translates directly to PILP. The process of generating theories also mimics 
ILP, since they are based on the logical clauses in the PBK. Therefore the search space algorithm of PILP has the same efficiency issues of ILP's. Furthermore, PILP adds an extra level of complexity due to the probabilistic evaluation of theories w.r.t. the examples. The background knowledge can be composed of (Horn) clauses, which can be facts or definite clauses Definite clauses are composed of a head and a body, and the body represents the explanation for the head. Facts and definite clauses' heads are examples of literals that ILP and PILP use to build rules.

As mentioned before, in this work, probabilities are annotated according to ProbLog's syntax [10]. Each clause $p_{j}:: c_{j}$ in the PBK represents an independent binary random variable in ProbLog, meaning that it can either be true with probability $p_{j}$ or false with probability $1-p_{j}$. Each set of possible choices over all clauses of the PBK represents a possible world $\omega_{i}$, where $\omega_{i}^{+}$is the set of clauses that are true in that particular world, and $\omega_{i}^{-}=\omega_{i} \backslash \omega_{i}^{+}$is the set of clauses that are false. Since these clauses have a probabilistic value, a ProbLog program defining a probabilistic distribution over the possible worlds can be formalized as shown in Equation 1. A ProbLog query $q$ is said to be true in all worlds $w^{q}$ where $w^{q} \vDash q$, and false in all other worlds. As such, the success probability of a query is given by the sum of the probabilities of all worlds where it is found to be true, as denoted in Equation 2

$$
P\left(\omega_{i}\right)=\prod_{c_{j} \in \omega_{i}^{+}} p_{j} \prod_{c_{j} \in \omega_{i}^{-}}\left(1-p_{j}\right) \quad(1) \quad P(q)=\sum_{\omega_{i} \models q} P\left(\omega_{i}\right)
$$

One important difference between ILP and PILP lies in the assessment of the fitness of theories - in PILP the loss function must be able to evaluate probabilistic inputs. As such, the aim of PILP systems is to find theories which most closely predict the value of the examples (also ranging between 0 and 1), or rather that minimize the error between predictions and the examples' values.

Theories can be formed either by a single rule (clause) or by a set of rules (where the clauses are mutually disjunctive). The length of a theory is equal to the number of rules it contains. In SkILL, theories can be combined using either the AND or the OR operation, which correspond to the logical conjunction and disjunction of the rules in the theories, respectively. In the case of the AND operation, only single rules (theories of length one) can be combined, and the result is another theory of length one (e.g. combining theories $t(X)$ :$p(X)$ and $t(X):-q(X, Y)$ using the AND operation would result in theory $t(X)$ :$p(X), q(X, Y){ }_{1}^{1}$ Conversely, theories of any length can be combined using the OR operation, and the resulting theory's length is equal to the sum of the lengths of the combined theories (e.g. combining theories of length one $t(X):-r(X)$ and $t(X):-s(X, Y)$ using the OR operation would result in theory $t(X):-r(X) ; s(X, Y)$ of length 2).

\footnotetext{
${ }^{1}$ The unification of variables between the literals is obtained from the specified language bias.
} 
SkILL's algorithm is composed of two main steps: (i) building theories of length one (single rules) using the AND operation, and (ii) building theories of length greater than one using the OR operation. In step (i), single rules of increasing number of literals are built from the mode declarations using the AND operation. Adding literals to a rule in conjunction makes the resulting rule more specific. Once all possible rules are built and evaluated, the algorithm proceeds to step (ii) using the OR operation to combine single rules (theories of length one) into theories of greater length, up to a maximum length. By adding rules to a theory in disjunction, the resulting theory becomes more general.

In order to assess a theory's fitness, its exact probabilistic value for each example must be computed, so that the theory is evaluated exactly. This process can be very time consuming, since the evaluation process must consider all possible worlds where the theory may be true. For a small number of facts in the PBK this is not a problem, but exact computation grows exponentially as the size of the PBK is increased. Consider the process of evaluating exactly the theory $t(X):-p(X), q(X, Y)$. ProbLog would need to compute all possible worlds for this theory in order to assess the overall error of the theory's predictions against the examples. Whether the theory is stored for further combinations or discarded after the evaluation stage, the system has already spent a considerable amount of time just to evaluate it.

To mitigate this problem, this work introduces the estimation pruning strategy, which can discard theories based on their previously evaluated subparts. For instance, suppose that theories $t(X):-p(X)$ and $t(X):-q(X, Y)$ had already been evaluated - in that case, it is possible to make an estimation of the value of $t(X)$ :- $p(X), q(X, Y)$ based on this information. Thus, estimation pruning consists of ruling out theories that have poor estimations and exactly evaluating theories that have good estimations. In SkILL, the decision on whether a theory is discarded is made based on one of two criteria: soft pruning or hard pruning. After the initial step of estimating the values for each example, the estimated value's usefulness is assessed according to one of these criteria. Note that the criteria are directly applicable to the estimated probabilistic values in lieu of the exact predictions of a theory. The combination is then pruned away if it is found to be useless. Conversely, if the combination is considered useful, then exact probabilistic evaluation is performed and the theory and its exact evaluation are saved for the next iteration.

\section{Estimation Pruning}

Estimation pruning consists of estimating the predictions of two theories combined based on the individual predictions of each theory. Estimation pruning excludes combinations of theories whose estimated predictions suggest that the resulting theory will be too specific (for the AND operation) or too general (for the OR operation). This process is somehow similar to the evaluation of theories in ILP. For instance, the more specific theory $t_{s}$ will not cover more positive examples than a more general theory $t_{g}$ and so it can be discarded. 
Table 1. Expressions used to calculate estimations

\begin{tabular}{c||c|c|c|c|c} 
Operation & minimum & maximum & center & independence & exclusion \\
\hline \hline AND & $\max (0, A+B-1)$ & $\min (A, B)$ & $\frac{1}{2}(\min (A, B)+\max (0, A+B-1))$ & $A \times B$ & $\max (0, A+B-1)$ \\
OR & $\max (A, B)$ & $\min (A+B, 1)$ & $\frac{1}{2}(\max (A, B)+\min (A+B, 1)$ & $A+B-A \times B$ & $\min (A+B, 1)$
\end{tabular}

In the PILP setting, the exact probabilistic evaluation of a theory corresponds to the weighted proportion of worlds where the theory is true. The probabilistic value for an example $e$ using a theory $t$ is given by determining in how many worlds (of all possible worlds in the PBK) $t(e)$ is true. The challenge in estimating the value of a probabilistic evaluation knowing the values of the theories being combined lies in the fact that the amount of overlapping of the sets of worlds corresponding to those two theories is unknown before evaluation. If two theories are mutually exclusive (or disjoint) w.r.t. the PBK, then their overlap is null. On the other hand, if a theory is more specific than another, the former will cover a subset of the worlds covered by the latter. Theories can also be independent, meaning that the probability that one theory is true in a world does not change the probability that another theory is also true in that world.

Despite this uncertainty, it is possible to calculate the interval where the predictions of a combination of two theories will be (this is depicted in Fig. 1 1 as a shaded area). The lower and upper bounds of the interval are determined by the predictions of the theories that are being combined $\left(t_{1}\right.$ and $t_{2}$ in Fig. 1(a)|r). Depending on where the resulting theory $t$ will lie in the interval, the (vertical) distance between $t^{\prime}$ s values and the example values (squares in Fig. 1) will vary, and as $t$ converges to the examples, its prediction quality is improved.

This work presents five estimators that can be used to estimate the value of theories, namely: minimum, maximum, center, independence and exclusion. These estimators predict different sets of values inside the estimation interval, based on different set theory cases. The minimum and maximum estimators correspond to the lower and upper boundaries of the estimation interval (min and max estimators in Fig. 1. respectively). The center estimator (ctr in Fig. 1) is the center of the estimation interval (halfway between minimum and maximum). The independence estimator (ind in Fig. 1) assumes that theories $t_{1}$ and $t_{2}$ are independent and calculates the values of their combination accordingly. The exclusion estimator (not depicted in Fig. 1) assumes that the theories $t_{1}$ and $t_{2}$ are as exclusive as possible. In the AND operation, the exclusion estimator is equal to the minimum estimator, since when two theories are mutually exclusive, their amount of overlap is minimum. The first row in Table 1 summarizes the expressions used to calculate these estimations.

After calculating an estimation for the combination of theories $t_{1}$ and $t_{2}$, it is necessary to decide, based on the estimation, whether the combination of theories should be evaluated. Thus, two pruning criteria can be used: hard pruning (Fig. 1(c) or soft pruning (Fig. 1(d)). For the AND operation, the hard pruning criterion discards theories that are too specific in any of their predictions. This

\footnotetext{
2 Theories are indexed only for clarity's sake. They correspond to the same concept to be learned.
} 


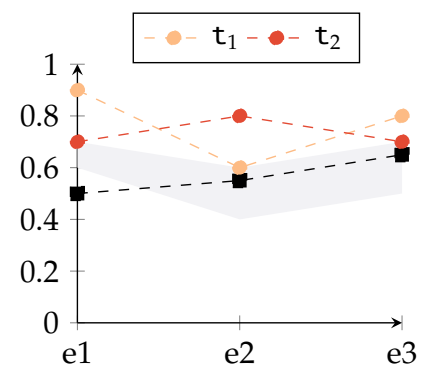

(a) Theories

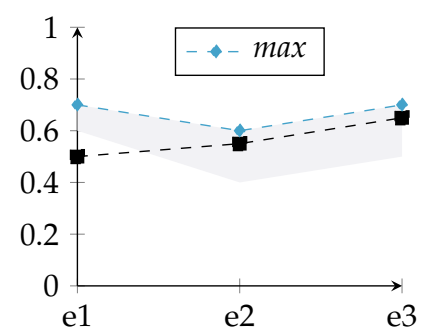

(c) Hard Pruning

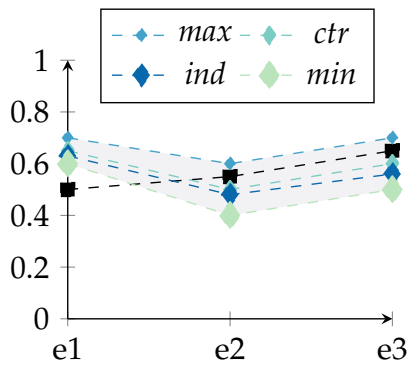

(b) Estimators

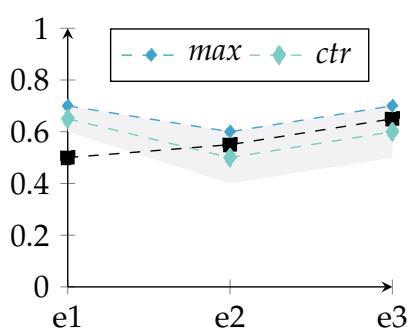

(d) Soft Pruning

Fig. 1. Estimators in AND operation. The x-axis contains three examples and the $y$-axis represents probabilistic values. Examples are depicted as squares, theories $t_{1}$ and $t_{2}$ as circles and estimators min, $\max , c t r$, ind as diamonds.

means that the estimations must be higher than the examples' values in every point (in Fig. 1(c) this only happens if the maximum estimator is being used to estimate the combination). The soft pruning criterion only prunes the theory away if it is overall more specific than the example values. In Fig. 1(d), the estimators that are not discarded are those that are above (maximum) or equally above and below (center) the examples' values. Estimator center is kept because its estimations are just a small distance below two example values but are a large distance above the first example value, which balances out. Pruning combinations of theories can be extended to the OR operation. Like the AND operation, this strategy estimates the value of a combination of two theories. In the OR setting, theories are excluded when they are found to be too general to benefit from further combination. Based on the expressions presented in Table 1 and following a similar reasoning to the AND operation, the same five estimators can be defined. Again, the minimum and maximum estimators define the estimation interval based on $t_{1}$ and $t_{2}$. The center estimator is the value halfway between the lower and upper boundaries of the estimation interval and the independence estimator assumes theories are independent. In the OR operation, the exclusion estimator is equal to the maximum estimator, because when the overlap of two theories is minimum (they are exclusive), the largest area is covered. When the exclusion estimator is used in both AND and OR operations, the result it pro- 
duces will be different than it would be using only the maximum or minimum estimators for both operations. For this reason, it is relevant to consider the exclusion estimator. The hard and soft pruning criteria can also be extended to the OR operation. The hard pruning criterion now excludes estimations that are too general in any point to be of interest. This translates to keeping only estimators whose values are always lower than or equal to the examples' values. Like the AND operation, the soft pruning criterion only discards estimators whose values are overall more general than the examples' values.

\section{Experiments}

The experiments presented in this section were run on a machine containing 4 AMD Opteron 6300 processors with 16 cores each and a total of 250GB of RAM. The metabolism dataset is an adaptation of the dataset originally from the 2001 KDD Cup Challeng $\AA^{3}$. The breast cancer dataset contains data from 130 biopsies dating from January 2006 to December 2011, which were prospectively given a non-definitive diagnosis at radiologic-histologic correlation conferences. The athletes dataset consists of a subset of facts regarding athletes and the sports they play collected by the never-ending language learner NELL $\mathrm{L}^{4}$. For the metabolism and athletes datasets, a number of n-times hold-out sets were made and all measurements were averaged out over the folds. In the breast cancer dataset, leave-one-out cross-validation was used.

Different combinations of estimation pruning were tested: only pruning the AND operation, only pruning the OR operation, and pruning both operations. The pruning settings are reported as a set of two letters: the first letter is the AND pruning option and the second is the OR pruning option. Pruning options can be soft pruning $(\mathrm{S})$, hard pruning $(\mathrm{H})$ or no pruning $(\mathrm{x})$. For example, using this codification, $\mathrm{xS}$ stands for no AND pruning and soft OR pruning. For each configuration, several measurements were recorded for each dataset: execution time, probabilistic accuracy on the test set, and number of rules and theories pruned. The probabilistic accuracy metric used in this work is equivalent to the mean absolute error of predictions calculated against example values, and was first introduced by De Raedt and Thon in [6].

Tables 2, 3 and 4 present the speedups and ratio of probabilistic accuracy for the metabolism, breast cancer and athletes datasets, respectively. The speedup $\frac{B_{t}}{P_{t}}$ is calculated w.r.t. the $B_{t}$ base case time (no pruning) for different $P_{t}$ pruning options' execution time. If there is a slowdown, the inverse speedup $\frac{P_{t}}{B_{t}}$ is presented as a negative number. The ratio of the probabilistic accuracy $\frac{P_{a}}{B_{a}}$ is calculated for each probabilistic settings $P_{a}$ w.r.t the probabilistic accuracy of the $B_{a}$ base case. Similarly to the speedup, when the probabilistic accuracy decreases, the inverse of the ratio is given $\frac{B_{a}}{P_{a}}$ as a negative number. Figures 2,3 and 4 depict the variation in execution time in minutes (left y-axis) and the variation in

3 http://www . cs . wisc. edu/ dpage/kddcup2001

4 http://rtw.ml.cmu.edu 
Table 2. Speedup and probabilistic accuracy ratio for metabolism dataset

\begin{tabular}{c||c|c|c|c|c|c}
\multicolumn{7}{c}{ Speedup } \\
Est & Sx & Hx & xS & xH & SS & HH \\
\hline \hline min & 1.45 & 1.47 & -1.03 & 1.36 & 1.47 & 2.52 \\
max & 1.56 & 1.56 & -1.09 & 1.94 & 1.61 & 5.66 \\
ctr & 1.57 & 1.58 & 1.03 & 1.95 & 1.61 & 5.65 \\
ind & 1.35 & 1.33 & -1.23 & 1.64 & 1.46 & 5.37 \\
exc & 1.57 & 1.58 & -1.04 & 1.95 & 1.58 & 5.69
\end{tabular}

Probabilistic Accuracy Ratio

\begin{tabular}{c||c|c|c|c|c|c} 
Est & $\mathrm{Sx}$ & $\mathrm{Hx}$ & $\mathrm{xS}$ & $\mathrm{xH}$ & $\mathrm{SS}$ & $\mathrm{HH}$ \\
\hline \hline min & 1.00 & 1.00 & 1.00 & 1.01 & 1.00 & 1.00 \\
$\max$ & 1.00 & 1.00 & 1.00 & 1.00 & 1.00 & -1.01 \\
ctr & 1.00 & 1.00 & 1.00 & 1.00 & -1.01 & -1.01 \\
ind & 1.00 & 1.00 & 1.00 & 1.00 & 1.00 & -1.01 \\
exc & 1.00 & 1.00 & 1.00 & 1.00 & 1.00 & -1.01
\end{tabular}

Table 3. Speedup and probabilistic accuracy ratio for breast cancer dataset

\begin{tabular}{c||c|c|c|c|c|c}
\multicolumn{7}{c}{ Speedup } \\
Est & Sx & Hx & $\mathrm{xS}$ & $\mathrm{xH}$ & $\mathrm{SS}$ & $\mathrm{HH}$ \\
\hline \hline min & 7.09 & 7.18 & 1.42 & 1.41 & 22.44 & 21.46 \\
max & 7.16 & 7.06 & 1.65 & 1.63 & 25.24 & 23.49 \\
ctr & 7.04 & 6.98 & 1.63 & 1.63 & 25.25 & 24.80 \\
ind & 7.20 & 7.02 & 1.42 & 1.29 & 22.62 & 22.84 \\
exc & 7.19 & 7.19 & 1.63 & 1.62 & 25.00 & 24.80
\end{tabular}

Probabilistic Accuracy Ratio

\begin{tabular}{c||c|c|c|c|c|c} 
Est & $\mathrm{Sx}$ & $\mathrm{Hx}$ & $\mathrm{xS}$ & $\mathrm{xH}$ & $\mathrm{SS}$ & $\mathrm{HH}$ \\
\hline \hline min & 1.09 & 1.09 & 1.00 & 1.00 & 1.09 & 1.09 \\
max & 1.09 & 1.09 & 1.00 & 1.00 & 1.09 & 1.09 \\
ctr & 1.09 & 1.09 & 1.00 & 1.00 & 1.09 & 1.09 \\
ind & 1.09 & 1.00 & 1.00 & 1.00 & 1.09 & 1.09 \\
exc & 1.09 & 1.09 & 1.00 & 1.00 & 1.09 & 1.09
\end{tabular}

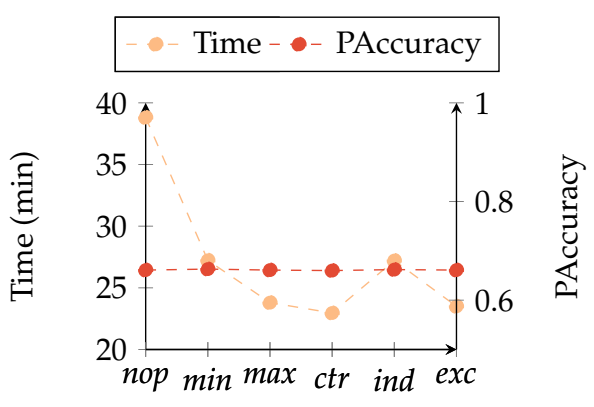

Fig. 2. Average time (in minutes) and probabilistic accuracy in metabolism dataset, for the base case (nop) and the five estimators. Values for each estimator are the average of its result over the pruning options.

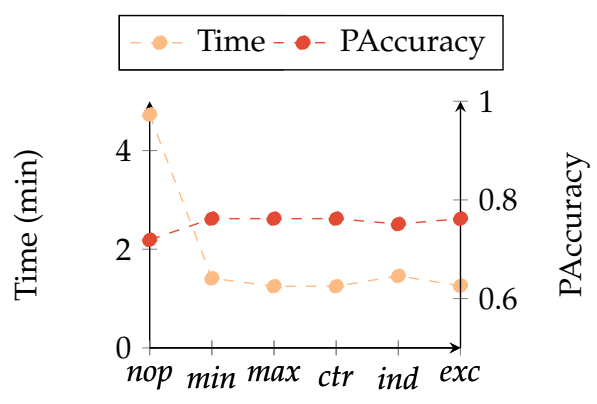

Fig. 3. Average time (in minutes) and probabilistic accuracy in breast cancer dataset, for the base case (nop) and the five estimators. Values for each estimator are the average of its result over the pruning options.

probabilistic accuracy (right y-axis) for all estimators in the metabolism, breast cancer and athletes datasets, respectively. The estimators analysed were the base case (no estimation pruning performed, or nop), minimum (min), maximum (max), center (ctr), independence (ind), and exclusion (exc). Each dataset's results will be discussed next.

For the metabolism dataset, results in Table 2 show that the greatest reduction in execution time is achieved by all estimators in the $\mathrm{HH}$ pruning setting. The $\mathrm{xS}$ pruning setting shows the slowest execution times with all estimators, except center, causing a slowdown. There is no significant reduction in proba- 
bilistic accuracy in any setting. Figure 2 shows that, overall, the probabilistic accuracy of the theories is unchanged and that the maximum, center and exclusion estimators can all reduce execution time from 40 to less than 25 minutes.

Results in Table 3 show that, in the breast cancer dataset, the greatest reduction in execution time can be achieved by using pruning in both the AND and the OR operations (SS and HH settings). The pruning settings that use only OR pruning ( $\mathrm{xS}$ and $\mathrm{xH})$ present more modest reductions of execution time (about 1.5 times) when compared to the settings that use only AND pruning (about 7 times). Although the OR operation has the potential to increase the (probabilistic) accuracy of true positives, it may also increase the accuracy of false positives. On the other hand, the AND operation, for this domain, works better, since it maintains the accuracy of true positives while decreasing the accuracy of false positives, when combining literals in a theory. The predictive accuracy of the best theory in this dataset never decreases, and in some settings (Sx, Hx, SS and HH in Table 3) even increases slightly. This effect is due to a reduction in overfitting caused by the exclusion of some theories that are better on the training set but perform worse on the test set. Figure 3 shows that, on average, the maximum, center and exclusion datasets can reduce execution time from over 4 minutes to about 1 minute.

In the athletes dataset, again the $\mathrm{HH}$ pruning setting can reduce most execution time. However, the reduction using estimators minimum and center is much less than that of estimators maximum, independence and exclusion, where the execution is about 50 times faster (Table 4). Estimators minimum and center are consistently slower in other pruning settings (xS, $\mathrm{xH}$ and SS), and the $\mathrm{xS}$ and $\mathrm{xH}$ settings present the lowest reduction in execution time in this dataset, of 2 times on average. Similarly to the other datasets, Table 4 shows that the probabilistic accuracy in the athletes dataset presents no significant reduction

Table 4. Speedup and probabilistic accuracy ratio for athletes dataset

\begin{tabular}{c||c|c|c|c|c|c}
\multicolumn{8}{c}{ Speedup } \\
Est & Sx & Hx & xS & xH & SS & HH \\
\hline \hline min & 3.34 & 3.33 & 1.01 & 1.66 & 3.63 & 9.48 \\
max & 3.35 & 3.35 & 2.12 & 2.19 & 12.40 & 49.72 \\
ctr & 3.20 & 3.28 & 1.00 & 1.80 & 3.62 & 19.82 \\
ind & 3.33 & 3.34 & 2.10 & 2.17 & 12.34 & 50.36 \\
exc & 3.31 & 3.23 & 2.02 & 2.11 & 11.86 & 48.01
\end{tabular}

Probabilistic Accuracy Ratio

\begin{tabular}{c||c|c|c|c|c|c} 
Est & $\mathrm{Sx}$ & $\mathrm{Hx}$ & $\mathrm{xS}$ & $\mathrm{xH}$ & $\mathrm{SS}$ & $\mathrm{HH}$ \\
\hline \hline min & -1.06 & -1.06 & 1.00 & 1.00 & -1.11 & 1.00 \\
max & -1.06 & -1.06 & 1.00 & 1.00 & -1.15 & 1.00 \\
ctr & -1.06 & -1.06 & 1.00 & 1.00 & -1.08 & 1.00 \\
ind & -1.06 & -1.06 & 1.00 & 1.00 & -1.15 & 1.00 \\
exc & -1.06 & -1.06 & 1.00 & 1.00 & -1.15 & 1.00
\end{tabular}

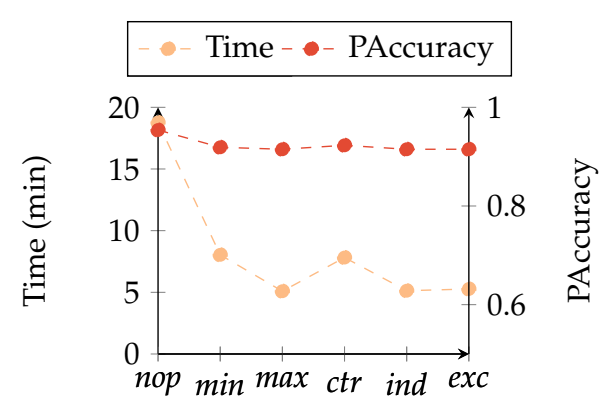

Fig. 4. Average time (in minutes) and probabilistic accuracy in athletes dataset, for the base case (nop) and the five estimators. Values for each estimator are the average of its result over the pruning options. 
Table 5. Number of single rules/theories evaluated for the athletes dataset

\begin{tabular}{c||c|c|c|c|c|c|c} 
Est & $\mathrm{xx}$ & $\mathrm{Sx}$ & $\mathrm{Hx}$ & $\mathrm{xS}$ & $\mathrm{xH}$ & $\mathrm{SS}$ & $\mathrm{HH}$ \\
\hline \hline min & $2414 / 1989$ & $164 / 968$ & $164 / 968$ & $2414 / 1981$ & $2414 / 604$ & $164 / 913$ & $164 / 361$ \\
$\max$ & $2414 / 1989$ & $164 / 968$ & $164 / 968$ & $2414 / 69$ & $2414 / 0$ & $164 / 243$ & $164 / 0$ \\
ctr & $2414 / 1989$ & $164 / 968$ & $164 / 968$ & $2414 / 1974$ & $2414 / 381$ & $164 / 907$ & $164 / 128$ \\
ind & $2414 / 1989$ & $164 / 968$ & $164 / 968$ & $2414 / 69$ & $2414 / 0$ & $164 / 243$ & $164 / 0$ \\
exc & $2414 / 1989$ & $164 / 968$ & $164 / 968$ & $2414 / 69$ & $2414 / 0$ & $164 / 243$ & $164 / 0$
\end{tabular}

and, in particular, in the $\mathrm{xS}, \mathrm{xH}$ and $\mathrm{HH}$ settings it is not reduced at all. Estimators maximum, independence and exclusion present the greatest overall reduction in execution time (Fig. 4), from 20 to about 5 minutes, on average.

Finally, for the athletes dataset, Table 5 presents the number of probabilistic evaluations performed for each pruning setting and estimator. The first number corresponds to single rules (theories of length one) evaluated, and thus the reduction is caused by AND pruning. Similarly, the second number in each cell is the number of theories of length greater than one, and its reduction is caused by OR pruning. The greatest reductions correspond to the HH setting (column 8 in Table 5, and are consistent with the settings in Table 4 that presents the greatest speedups. Additionally, from Figure 4 the three fastest estimators (in average) are also the estimators that in Table 5 prune away most theories. In particular, the number of theories pruned away during OR pruning is significantly lower for estimators max, ind and exc when compared to estimators min and ctr. The same trend can be observed in the other datasets but results were omitted due to lack of space.

\section{Conclusion}

This work proposed five PILP estimators whose aim is to alleviate the overhead imposed by the exact evaluation of combinations of candidate probabilistic theories. Because PILP theories can be built using both conjunction (AND operation) and disjunction (OR operation), the estimators must be adapted accordingly. The estimators were implemented in the estimation pruning stage of the SkILL system, but can be generalized to any PILP engine. Results showed that all estimators resulted in faster execution times when coupled with an $\mathrm{H}$ (hard) pruning setting and, in particular, the $\mathrm{HH}$ pruning setting showed the greatest speedups and also the greatest reduction in the number of probabilistic evaluations performed. Even though all estimators maintain predictive quality and reduce execution time, estimators maximum and exclusion are overall faster, and opting for one of these estimators in lieu of estimators minimum, center or independence can result in an up to 5 times faster runtime for the same pruning setting. Future work includes adding an estimator that divides the estimation interval according to a user-defined distance and dynamically adapting the estimator setting during runtime. It also seems relevant to compare against different learning systems with a number of probabilistically annotated datasets in order to assess the quality of the models and execution time. 


\section{Acknowledgments}

Joana Côrte-Real is funded by the FCT grant SFRH/BD/52235/2013. This work is partially funded by the ERDF through the COMPETE 2020 Programme within project POCI-01-0145-FEDER-006961, by National Funds through the FCT as part of project UID/EEA/50014/2013, and by the North Portugal Regional Operational Programme, under the PORTUGAL 2020 Partnership Agreement, and through the European Regional Development Fund as part of project NanoSTIMA (NORTE-01-0145-FEDER-000016).

\section{References}

1. E. Bellodi and F. Riguzzi. Structure learning of probabilistic logic programs by searching the clause space. Theory and Practice of Logic Programming, 15(02).

2. J. Côrte-Real, T. Mantadelis, I. Dutra, R. Rocha, and E. Burnside. SkILL - a Stochastic Inductive Logic Learner. In Proceedings of the 14th International Conference on Machine Learning and Applications, pages 555-558. IEEE, 2015.

3. L. De Raedt, A. Dries, I. Thon, G. Van den Broeck, and M. Verbeke. Inducing Probabilistic Relational Rules from Probabilistic Examples. In International Joint Conference on Artificial Intelligence, pages 1835-1843. AAAI Press, 2015.

4. L. De Raedt and K. Kersting. Probabilistic inductive logic programming. In International Conference on Algorithmic Learning Theory, pages 19-36. Springer, 2004.

5. L. De Raedt and A. Kimmig. Probabilistic (logic) programming concepts. Machine Learning, 100(1):5-47, 2015.

6. L. De Raedt and I. Thon. Probabilistic Rule Learning. In Inductive Logic Programming, pages 47-58. Springer, 2011.

7. L. Getoor. Introduction to Statistical Relational Learning. MIT press, 2007.

8. L. Getoor, B. Taskar, and D. Koller. Selectivity Estimation using Probabilistic Models. In ACM SIGMOD Record, volume 30, pages 461-472. ACM, 2001.

9. K. Kersting, L. De Raedt, and S. Kramer. Interpreting Bayesian Logic Programs. In AAAI Workshop on Learning Statistical Models from Relational Data, pages 29-35, 2000.

10. A. Kimmig, B. Demoen, L. De Raedt, V. Santos Costa, and R. Rocha. On the Implementation of the Probabilistic Logic Programming Language ProbLog. Theory and Practice of Logic Programming, 11(2 \& 3):235-262, 2011.

11. S. Kok and P. Domingos. Learning the Structure of Markov Logic Networks. In International Conference on Machine learning, pages 441-448. ACM, 2005.

12. S. Muggleton. Stochastic Logic Programs. Advances in inductive logic programming, 32:254-264, 1996.

13. S. Natarajan, T. Khot, K. Kersting, B. Gutmann, and J. Shavlik. Gradient-based boosting for statistical relational learning: The relational dependency network case. Machine Learning, 86(1):25-56, 2012.

14. M. Richardson and P. Domingos. Markov Logic Networks. Machine learning, 62(12):107-136, 2006.

15. V. Santos Costa, D. Page, M. Qazi, and J. Cussens. CLP(BN): Constraint Logic Programming for Probabilistic Knowledge. In Conference on Uncertainty in Artificial Intelligence, pages 517-524, 2002.

16. T. Sato and Y. Kameya. PRISM: A language for symbolic-statistical modeling. In International Joint Conference on Artificial Intelligence, volume 97, pages 1330-1339. Morgan Kaufmann, 1997.

17. O. Schulte, H. Khosravi, A. Kirkpatrick, T. Gao, and Y. Zhu. Modelling relational statistics with Bayes Nets. Machine Learning, 94(1):105-125, 2014. 